\title{
Multi-center semi-empirical quantum models for carbon under extreme thermodynamic conditions
}

\author{
Nir Goldman ${ }^{\mathrm{a}, *}$ \\ ${ }^{a}$ Physical and Life Sciences Directorate, Materials Sciences Division, Lawrence \\ Livermore National Laboratory, Livermore, California 94550
}

\begin{abstract}
We report on the development of many-body density functional tight binding (DFTB) models for carbon, which include either explicit or implicit calculation of multi-center terms in the Hamiltonian. We show that all of our methods yield accurate eigenstates and eigenfunctions for both ambient diamond and transitions to molten, metallic states. We then determine a three-body repulsive energy to compute accurate equation of state and structural properties for carbon under these conditions. Our results indicate a straightforward approach by which many-body effects can be included in DFTB, thus extending the method to a wide variety of systems and thermodynamic conditions.
\end{abstract}

\section{Introduction}

The equation of state of materials under extreme pressures and temperatures $(\mathrm{P}>10 \mathrm{GPa}, \mathrm{T}>1000 \mathrm{~K} ; 1 \mathrm{GPa}=10 \mathrm{kbar})$ is of great importance for understanding planetary interiors and the chemical reactivity that occurs under strong dynamic compression. Knowledge of the high pressuretemperature properties of simple compounds such as solid phases of carbon is needed to devise models of Neptune and Uranus[1], brown dwarfs[2], and extra-solar carbon planets[3]. In addition, carbon is a major component of candidate materials for the design of fusion capsules for the National Ignition Facility (NIF)[4]. Late-time soot condensation is known to account for significant energy release in detonating carbon-rich energetic materials[5]. Similar questions arise in origin of life studies, where the creation of organic

\footnotetext{
${ }^{*}$ Corresponding author

Email address: ngoldman@llnl.gov (Nir Goldman) 
nanoparticles is predicted to be a crucial step in the impact synthesis of amino acids[6, 7] and possible precursors to DNA/RNA nucleotides[8].

Diamond anvil cell experiments have successfully accessed high pressure, low temperature states of matter [9], as well as the lower pressure, high temperature melting lines of compressed materials[10]. Laser-driven ramp compression[11] and shock compression experiments[12-14] have elucidated many high pressure properties of carbon, including the transition to a conducting liquid and indirect observation of exotic solid phases[15, 16]. Experiments tend to rely on equation of state (EOS) models for interpretation, which have been shown to be inaccurate for some systems $[17,18]$ and often neglect chemical kinetics that can affect measurements[18]. Determination of experimental temperatures generally remains an unresolved issue, partially due to large uncertainties in the calibration of pyrometric measurements[1921]. In addition, carbon has been computed to have a number of high pressure metastable states with high energetic barriers[22]. These states could be accessed during many compression experiments, whereas EOS models predict equilibrium states of matter over essentially infinite time scales[23].

Molecular Dynamics (MD) simulations provide an independent route to time resolved phase transformations and chemical reactivity determination, where material properties such as phase diagrams and reaction pathways are readily computed. Accurate modeling of the breaking and forming of bonds in condensed phases frequently requires the determination of many nuclear-body effects[24, 25], which are included in quantum theories such as Density Functional Theory (DFT) [26, 27]. DFT remains one of the most popular and widely used modeling methods in condensed matter physics, computational chemistry, and materials science for prediction of material properties and chemical reactivity. DFT has been shown to accurately reproduce the phase boundaries and thermal decomposition of many materials[10, 28, 29], particularly at higher thermodynamic conditions like planetary interiors[30-32], where long-range effects like dispersion are less important. DFT-MD simulations, however, scale poorly with computational effort and thus are generally limited to system sizes of hundreds of atoms and time-scales of 10 's of picoseconds[6, 31-34]. In contrast, chemical equilibrium can occur over nanosecond timescales or greater in many experiments[11, 12, 14, 16, 18, 21]. Empirical models, though highly computationally efficient, tend to perform poorly for materials and thermodynamic properties outside of their training set, and in general cannot capture the thermal electronic excitations that promote chemical reactivity and soften pressures under hot, dense conditions[35, 36]. As a result, a computationally efficient and yet accurate quantum chemical method would aid in direct 
modeling and interpretation of high pressure-temperature experiments on many of these materials.

The density functional tight binding method (DFTB) holds promise as an alternate approach for simulations of reactive materials. DFTB is an approximate quantum simulation technique whereby the Hamiltonian from Kohn-Sham DFT is expanded about a neutral reference density and is taken to be two-center, only (discussed below). As a result, the majority of the electronic interactions are pre-computed and mapped to a radial grid. Calculation of the electron states is combined with a pre-determined empirical repulsive energy, allowing for several orders of magnitude increase in computational efficiency while retaining most of the accuracy of DFT[37]. This allows DFTB simulations to achieve close to equilibrium timescales for reactivity under extreme conditions (e.g., up to a nanosecond), whereas standard DFT calculations are generally very far from equilibrium due to their computational expense. Most implementations of DFTB utilize a minimal basis set (e.g., s and p-orbitals for carbon). DFTB has been shown to yield accurate results for energetic materials at relatively low conditions $(\mathrm{P}<100 \mathrm{GPa}, \mathrm{T}$ $<4000 \mathrm{~K})[5,38]$. Nonetheless, DFTB suffers from three separate limitations that prevent accurate descriptions of many reactive systems of interest:

- For conditions such as electron delocalization and bond dissociation in the condensed phase, inclusion of crystal-field-like (many-body) effects on the total energy are needed in order yield accurate bonding energies and band structures[39-42]. These higher-order terms are explicitly neglected in the DFTB Hamiltonian. The electronic density of diamond compared to a metallic liquid state at hot, compressed conditions indicates that significant electron delocalization occurs for this transition (Fig. 1). Many-body interactions account for $\sim 20 \%$ of the total energy for diamond under pressures greater than $2000 \mathrm{GPa}[43]$. Previous work has shown that the vast majority of the interatomic energy is contained within the sum of the two and three-body terms for a broad range of materials[25, 40, 44], including insulator to metal transitions at high pressures and temperatures[43].

- The limited basis set is too small for many applications. DFT calculations of binding energies require expanded basis sets in order to achieve chemical accuracy, e.g., Ref. [45]. Reactive conditions can yield highly unique bonding arrangements of compounds and ionic species that require larger basis sets for accurate computation[31, 32, 46].

- The repulsive energy can be non-pairwise for reactive conditions[47]. 
This can be the case at both high thermodynamic conditions and for transition states in condensed phases at ambient conditions, where a number of different atomic-centers can interact simultaneously.

All of the above issues are likely to be enhanced at extreme conditions, as interatomic distances become relatively short, and elevated temperatures and pressures can induce some degree of thermal electronic excitation. Attempts have been made to improve upon the two-center tight-binding approach through both explicit calculation of three-center Hamiltonian terms[39-41] and inclusion of an environmental dependence of matrix elements in the form of electrostatic screening[42, 48, 49]. These efforts have not entered into mainstream use, either because explicit three-center approaches are too computationally cumbersome and memory intensive[44], or because overly simplistic screening models are parameterized to fit bulk data for specific phases and conditions and require re-parameterization for each new system to be studied[42, 49,50]. A DFTB method that computes third-order charge interactions between atomic sites has been developed[51, 52], but does not explicitly include greater than two-center terms in the Hamiltonian.

Recently, Goldman, et al., rectified some of these issues by developing DFTB models (named DFTB-p3b) that retain the two-center Hamiltonian approximation but included an extended, polarizable basis set (e.g., up to dorbitals for carbon) along with a three-body, environmentally dependent repulsive potential[43, 53, 54]. The three-body repulsive energy was necessary in order to account for the greater than two-body forces due to electron delocalization present under molten conditions. The DFTB-p3b models provide an improved description of the electronic states of the metallic liquid phases of both carbon and hydrogen, as well as accurate shock Hugoniot curves and radial distribution functions (RDFs), while allowing for two-orders of magnitude increased computational efficiency over standard DFT calculations. However, results indicated a "spike" in the electronic density of states at high energy for some high pressure carbon systems[43], as well as a manifold of electronic states that was narrow in comparison to converged DFT calculations $[43,53]$.

In this work, we present three different methods for overcoming these errors by including many-body effects in the DFTB Hamiltonian. We first provide an overview of DFTB and then describe a method for explicitly including these effects in the Hamiltonian matrix by using results from DFT calculations on small atomic clusters. We develop this explicit type of model for a minimal basis set for carbon containing up to p-orbitals, as well as expanded basis set containing up to d-orbitals. We also present an implicit 
N-body method which retains the same approximate two-center Hamiltonian of standard DFTB, but implicitly contains knowledge of the condensed phase in the diagonal part of the matrix. We then analyze both the electronic states and eigenfunctions from each method and observe that all three methods yield good agreement with DFT for the manifold of occupied electronic states, but that the implicit N-body method yields the best agreement with DFT for a description of the resulting wavefunctions. We then use a three-body parameterization of the repulsive energy for the implicit N-body method to yield accurate properties of high pressure diamond as well as the metallic liquid phase.

\section{Theoretical Background}

The derivation of DFTB from Kohn-Sham DFT has been discussed in detail in the literature[37, 55, 56]. Here, we briefly derive the equations for DFTB, with emphasis on the final expressions for the total energy. The Kohn-Sham DFT energy can be written as follows:

$$
\begin{aligned}
E[n]= & \sum_{a} f_{a}\left\langle\psi_{a}\left|\left(-\frac{1}{2} \nabla^{2}+\int V_{e x t}(\mathbf{r}) n(\mathbf{r})+\frac{1}{2} \int^{\prime} \frac{n\left(\mathbf{r}^{\prime}\right) \mathrm{d}^{3} r^{\prime}}{\left|\mathbf{r}-\mathbf{r}^{\prime}\right|}\right)\right| \psi_{a}\right\rangle \\
& +E_{x c}[n(\mathbf{r})]+E_{I I} .
\end{aligned}
$$

Here, $f_{a}$ is the Fermi occupation of the single-particle eigenstate $\psi_{a}, n(\mathbf{r})$ is the electron density of the system, $V_{\text {ext }}(\mathbf{r})$ is the external potential, including electron-ion interactions, $E_{x c}$ is the exchange-correlation energy, and $E_{I I}$ is the ion-ion repulsion energy. For brevity, we use the following notation: $\int \mathrm{d}^{3} r \rightarrow \int, \int \mathrm{d}^{3} r^{\prime} \rightarrow \int^{\prime}, n(\mathrm{r}) \rightarrow n$, and $n\left(\mathrm{r}^{\prime}\right) \rightarrow n^{\prime}$.

We now assume a neutral, spherical charge density $n_{0}$ that neighbors the true density of the system, $n(\mathrm{r})=n_{0}(\mathrm{r})+\delta n$, where $\delta n$ is supposed to be small. Expanding $E[n]$ to second order in fluctuation yields the following expression:

$$
\begin{aligned}
E[\delta n] \approx & \sum_{a} f_{a}\left\langle\psi_{a}\left|-\frac{1}{2} \nabla^{2}+V_{e x t}+\int^{\prime} \frac{n_{0}^{\prime}}{\left|\mathbf{r}-\mathbf{r}^{\prime}\right|}+V_{x c}\left[n_{0}\right]\right| \psi_{a}\right\rangle \\
& +\frac{1}{2} \iint^{\prime}\left(\frac{\delta^{2} E_{x c}\left[n_{0}\right]}{\delta n \delta n^{\prime}}+\frac{1}{\left|\mathbf{r}-\mathbf{r}^{\prime}\right|}\right) \delta n \delta n^{\prime} \\
& -\frac{1}{2} \int \frac{n_{0}^{\prime} n_{0}}{\left|\mathbf{r}-\mathbf{r}^{\prime}\right|}+E_{x c}\left[n_{0}\right]-\int V_{x c}\left[n_{0}\right] n_{0}+E_{I I},
\end{aligned}
$$


where terms linear in $\delta n$ vanish. The first line in Equation 2 is the band structure energy:

$$
E_{B S}\left[n_{0}\right]=\sum_{a} f_{a}\left\langle\psi_{a}\left|H\left[n_{0}\right]\right| \psi_{a}\right\rangle,
$$

where the Hamiltonian $H$ does not contain any charge transfer terms. The second line from Equation 2 is the energy from charge fluctuations:

$$
E_{\text {coul }}=+\frac{1}{2} \iint^{\prime}\left(\frac{\delta^{2} E_{x c}\left[n_{0}\right]}{\delta n \delta n^{\prime}}+\frac{1}{\left|\mathbf{r}-\mathbf{r}^{\prime}\right|}\right) \delta n \delta n^{\prime} .
$$

$E_{\text {coul }}$ is mainly Coulombic can be solved for self-consistently, the details of which are discussed elsewhere[37, 56]. The last line in Equation 2 is called the repulsive energy:

$$
E_{r e p}=-\frac{1}{2} \int \frac{n_{0}^{\prime} n_{0}}{\left|\mathbf{r}-\mathbf{r}^{\prime}\right|}+E_{x c}\left[n_{0}\right]-\int V_{x c}\left[n_{0}\right] n_{0}+E_{I I},
$$

due to ion-ion repulsion term $E_{I I}$, though it also contains the Hartree (electrostatic potential) double-counting term as well as additional exchangecorrelation interactions.

For standard DFTB, $E_{B S}$ is computed using a minimal, non-orthogonal atomic basis set, made compact through application of a confining potential. The non-orthogonality of the basis set requires computation of the two-center overlap matrix. The Hamiltonian matrix is determined through two important approximations: (1) a one-center approximation is made for diagonal matrix elements, i.e., "onsite" terms, which are taken to be the orbital energies for a free neutral atom, and (2) a two-center approximation is made for off-diagonal matrix elements, i.e., "offsite" terms, or hopping terms[57, 58]. As a result, the Hamiltonian matrix elements can be written as follows:

$$
H_{\mu \nu}^{A B}= \begin{cases}E_{\mu \mu}^{A A} & \text { if } A=B, \mu=\nu \\ \left\langle\phi_{\mu}^{A}\left|\hat{T}+V_{0}\left(n_{A}+n_{B}\right)\right| \phi_{\nu}^{B}\right\rangle & \text { if } A \neq B, \mu \neq \nu \\ 0 & \text { otherwise }\end{cases}
$$

Here, the $A$ and $B$ indices correspond to the atomic sites, $\mu$ and $\nu$ to atomic orbitals on each atom, respectively, of specific angular momentum, and $E_{\mu \mu}^{A A}$ is the orbital energy in question. $V_{0}$ represents the external, Hartree, and exchange-correlation potentials for the $n_{0}$ reference densities. Offsite terms explicitly exclude three-center interactions, i.e., crystal-fieldlike terms $[56,58]$. These approximations allow for all matrix elements to 
be pre-computed on a radial grid. $E_{r e p}$ is generally determined by optimizing a pair-wise empirical function to either high-level quantum calculations of atomic cluster interactions or experimental results for gas-phase systems $[37,56,57]$. DFTB subsequently yields several orders of magnitude increase in computational efficiency over most DFT implementations, which project the electronic density onto a fine grid, and then use Fourier transforms to compute the Hartree potential and other terms.

\subsection{Explicit calculation of three-center matrix elements}

We first expand upon the DFTB approach by estimating the crystal-fieldlike terms that are explicitly neglected in its Hamiltonian. We input radial grids of our basis set from DFTB into the Siesta $a b$ initio molecular dynamics package[59], which allows for user input of a numerical atomic basis set. This capability allows for calculation of specific, non-approximated DFT matrix elements regardless of the basis set or confining potential used. For this work, all Siesta DFT calculations were performed with a Troullier-Martins pseuodopotential[60] and the Perdew-Burke-Ernzerhof generalized gradient approximation functional (PBE) for the exchange-correlation energy[61]. We note that our DFTB matrix elements were computed with the PBE functional as well. We then used Siesta to compute the Hamiltonian matrix elements for an assortment of carbon dimer and trimer geometries (Fig. 2). Atoms were oriented so as to easily decompose the Hamiltonian into different Slater-Koster interactions (e.g., $\operatorname{ss} \sigma, \operatorname{sp} \sigma, \operatorname{pp} \sigma, \operatorname{pp} \pi$, etc.). In doing so, we are able to estimate the effects of a neighboring atom in close proximity on the onsite and offsite parts of the Hamiltonian matrix. For the results reported here, $R_{A B}$ of the trimer was held fixed at $1.55 \AA$ (the diamond nearest-neighbor distance) in order to simplify the set of geometries to be sampled. The angular dependence of offsite interactions between higher angular momentum orbitals (particularly d-orbitals) has been observed in transition metal materials[62, 63], and was not sampled with the high-symmetry trimer geometries in our study. However, these effects appear to average out for the disordered metallic systems studied here.

We have computed the onsite and offsite matrix elements as a function of interatomic distance (Fig. 3). Our results indicate that in general, both onsite and offsite terms increase or decrease monotonically and smoothly as a function of increasing proximity of a neighboring atom. The sole exception was the $\mathrm{d} d \sigma$ offsite interaction, which had a more oscillatory character than the other terms likely due to its stronger angular character. In this way, the changes in the two-center onsite and three-center offsite terms from DFT can then be mapped onto radial functions for efficient calculation of 
an "environmentally-dependent" DFTB Hamiltonian. We can now include many-body effects in the electron state calculations without fitting to any bulk or condensed phase properties of specific materials. Calculating these effects through radial functions will not noticeably increase the computational wall clock time-to-solution, which is largely dominated by the matrix diagonalization step (over $70 \%$ of the CPU time for this study).

For our study, we adopt an approach that is similar to previous screening efforts[42, 49]. Our method represents a significant departure from these techniques in that the environmental dependence of each onsite and SlaterKoster offsite matrix element is computed individually from DFT, rather than by simultaneously fitting screening parameters for all matrix elements and the repulsive energy to specific condensed phase data. In addition, our approach allows for the matrix elements to increase or decrease from their DFTB values as needed, rather than forcing all of them to approach zero as a function of increasing density as in standard screening methods.

Empirical two-center functions can be used to independently map the environmental dependence of each onsite matrix energy,

$$
H_{\mu \mu}^{A A}=E_{\mu \mu}^{A A}\left[1-\sum_{B \neq A} F\left(R_{A B}\right)\right] .
$$

$E_{\mu \mu}^{A A}$ is once again the gas phase energy for orbital $\mu$ of atom $A$, and $F\left(R_{A B}\right)$ is a pair-wise function representing the change in the orbital energy as a function of the distance to a neighboring atomic site. In our study, we have found that the environmental dependence of the onsite matrix elements of $\mathrm{s}$, $\mathrm{p}$, and d-orbitals can be well represented by a sixth-order polynomial of the form:

$$
F\left(R_{A B}\right)=\sum_{i=2}^{6} \alpha_{i}\left(R_{c u t}-R_{A B}\right)^{i} .
$$

The cutoff radius $R_{\text {cut }}$ guarantees that the value of the function and its first derivative will have smooth behavior at the cutoff and will be equal to zero beyond. For this work, we have set the value of $R_{\text {cut }}$ to $1.55 \AA$. The coefficients $\alpha_{i}$ were then optimized to yield the correct relative change in each separate onsite term, determined from our computed DFT matrix elements (Table 1). Similar to previous efforts[43], the d-orbital energy was determined empirically in order to yield a band gap of ambient diamond and density of electronic states of the metallic liquid similar to results from DFT (Table 2). In our study, we found that applying an additional scaling 
term of 0.6 to $F\left(R_{A B}\right)$ yielded small improvements in the agreement with DFT results, particularly for the width of the electronic density of states at extremely high pressures and temperatures.

We have modeled the three-center offsite matrix elements according to the following functional form:

$$
\begin{aligned}
H_{\mu \nu}^{A B} & =\left\langle\phi_{\mu}^{A}\left|\hat{T}+V_{0}\left(\rho_{A}+\rho_{B}\right)\right| \phi_{\nu}^{B}\right\rangle\left[1+\Theta_{A B C}\left(R_{A B}, R_{B C}, R_{A C}\right)\right] \\
\Theta_{A B C}\left(R_{A B}, R_{B C}, R_{A C}\right) & =\tanh \left(\beta_{1} \sum_{C \notin\{A, B\}} \exp \left(-\beta_{2}\left[\frac{R_{A C}+R_{B C}}{R_{A B}}\right]\right)^{\beta_{3}}\right) .
\end{aligned}
$$

Here, $H_{\mu \nu}^{A B}$ is the standard Slater-Koster offsite matrix element of the DFTB two-center Hamitlonian, and the function $\Theta_{A B C}$ models its relative change as a function of three-body distances. Similar to the onsite matrix elements, the $\beta_{1}, \beta_{2}$, and $\beta_{3}$ terms were optimized separately for each individual SlaterKoster interaction (Table 3 ). In this case, improved accuracy for the electronic states of the metallic liquid was achieved by additional manual tuning of the three-center parameters for the $\operatorname{dd}(\sigma, \pi, \delta)$ interactions, the results of which are presented here.

\subsection{Implicit $N$-body method}

In our calculations, we have found that the environmental dependence of the onsite matrix elements varies much more strongly than the offsite terms. For example, the p-orbital onsite energy decreases by $\sim 200 \%$ at short interatomic distances, whereas the $\mathrm{pp} \pi$ offsite term decreases by only $\sim 15 \%$ as a third atomic site approaches distances of $1.5 \AA$, e.g., the approximate covalent bond length in ambient diamond (Fig. 3). We also note that at diamond covalent bond lengths, the s and p-orbital onsite energies are somewhat higher in value than their computed gas-phase values, i.e., $\sim 6 \%$ higher for the s-orbital and $\sim 30 \%$ for the p-orbital. In order to create a simple model for these changes, we have modified the orbital energies to correspond to values more likely found in the condensed phases. In doing so, we hope to implicitly include some of the greater than two-center contributions to the electronic states while retaining the simple one-center values for the onsite matrix elements and two-center offsite matrix elements expression for the Hamiltonian in Equation 6. Additional many-body effects needed for accurate determination of the forces can subsequently be computed in the repulsive energy. This implicit method thus can leverage the computational infrastructure of current DFTB implementations by only requiring modified 
inputs for the onsite energies, while potentially yielding a more accurate description of the electron states in condensed phases. For this work, we have chosen s and p-orbital energies that correspond to values computed from DFT at dimer distances equal to the diamond nearest neighbor distance (Table 2). Once again, the d-orbital energy was tuned to yield optimum results for the electronic density of states.

\section{Results and Discussion}

All of our calculations (three-body and implicit N-body) were performed with a modified version of the DFTB + code[64], with fractional electron occupancies calculated using the Mermin functional[65]. Previous results for carbon have shown that self-consistent charge (SCC) and non-SCC calculations yielded virtually identical results for both equation of state and structural properties such as radial distribution functions for extreme thermodynamic conditions[43, 66]. Here, we report on results from non-SCC calculations, only. We have examined our explicit three-body method by performing calculations with the normal minimal p-orbital basis set for carbon (labeled 3b-p) as well as with the extended d-orbital basis set (3b-d). DFT calculations were performed using the same p-orbital basis set (labeled DFT$\mathrm{SZ}$, for single- $\zeta$ ) and d-orbital basis set (DFT-SZP, for single- $\zeta$-polarizable). A single- $\zeta$ basis set is known to yield too high pressures for metallic liquid carbon relative to calculations with larger basis sets due to the six-fold coordination that can occur[43]. However, these calculations can still yield a reasonable description of the electronic states (discussed below), which could be coupled with a specific repulsive energy optimization to yield highly efficient DFTB calculations with accurate results for many physical properties. Our implicit N-body method showed marginal improvement for the p-orbital basis set, so here we report on results using the d-orbital basis set, only.

In order to assess the accuracy of the electronic states from each approach, we have computed the total electronic density of states (DOS) for both ambient diamond as well as metallic liquid carbon at high pressure and temperature. The DOS from our tight-binding calculations are independent of $E_{\text {rep }}$, allowing for a direct comparison to results from DFT without any empirical function fitting. The DOS for ambient diamond from all methods compare well to those from DFT (Fig. 4). This is expected in part since the environmental dependence of the Hamiltonian is fairly short-range, and greater than two-body effects in condensed phases of carbon are only significant at high pressure[43]. The 3b-p model predicts a larger band gap than DFT, but the $3 \mathrm{~b}-\mathrm{d}$ and implicit N-body methods show closer agreement. 
Results for the metallic liquid from our p-orbital calculations show that the 3b-p model yields a significantly improved DOS compared to standard DFTB (Fig. 5). In particular, the 3b-p model does not exhibit the spike in the DOS present for standard DFTB at approximately $-20 \mathrm{eV}$. The results compare well to those from DFT-SZ up to $\sim 5 \mathrm{eV}$, after which the $3 \mathrm{~b}-\mathrm{p}$ DOS begins to decrease monotonically. We note that no electronic states are occupied for this system at this high energy, even when taking into account thermal electronic excitations. Results for the d-orbital calculations show that both the implicit N-body and 3b-d calculations show good agreement with the DOS from DFT-SZP. The implicit N-body method shows somewhat closer agreement at around the Fermi energy (the total chemical potential for electrons) and at energies above $10 \mathrm{eV}$, whereas 3b-d shows improved agreement for the low-lying states below $-30 \mathrm{eV}$, which mostly have s-orbital character. However, both the $3 \mathrm{~b}-\mathrm{d}$ and implicit N-body results remove the spike in the DOS at $\sim 20 \mathrm{eV}$ that was computed from our previous DFTB-p3b model. In addition, both DFT-SZ and DFT-SZP yield very similar DOS results up to high energy $(\sim 20 \mathrm{eV})$. This is despite the higher than normal coordination of the carbon atoms at these conditions[43], which is facilitated through d-orbital bondings.

The accuracy of the wavefunctions from our methods can be assessed by determining their degree of delocalization around the Fermi energy. This can be determined through calculation of the inverse participation ratio (IPR)[67]:

$$
I\left(\psi_{n}\right)=\frac{N \sum_{i=1}^{N}\left(a_{n i}^{*} a_{n i}\right)^{2}}{\left(\sum_{i=1}^{N} a_{n i}^{*} a_{n i}\right)^{2}},
$$

Here, $\psi_{n}=\sum_{i=1}^{N} a_{n i} \phi_{i}$ is the $n$th eigenvector of the system and $\phi_{i}$ is one of the $N$ orbitals in our basis set. The IPR for an ideally delocalized state is around 1, whereas an ideally localized state (on one basis orbital) would yield $I=N$. Results from all of our methods agree reasonably well with those from DFT (Fig. 6). The IPR from the 3b-p calculation shows good agreement with that from DFT-SZ, indicating that our method yields a fairly faithful representation of the delocalization effects using a minimal basis set for this system. However, both 3b-p and DFT-SZ indicate more delocalized states than those from DFT-SZP, due in part to the increased energetics of forming six-fold coordinated states with a p-orbital basis set. For the dorbital calculations, the implicit N-body results shows better comparison to 
DFT-SZP than that from the 3b-d method up to the Fermi energy. Beyond the Fermi energy, the implicit N-body method states are too delocalized, and the $3 \mathrm{~b}-\mathrm{d}$ method exhibits better comparison to the result from DFT-SZP. Regardless, it is difficult a priori to ascertain how these differences in the IPR above the Fermi energy would manifest in terms of measurable physical properties. Accuracy of each method could be probed more directly through calculation of excited state properties (e.g., optical conductivities[68]), which is beyond the scope of this study.

Our results indicate that all three many-body methods presented here yield improved representations for both the electron states and wavefunctions for an electronically delocalized system. The implicit N-body approach holds an advantage over the 3b-p and 3b-d methods in terms of its simplicity and ease of implementation into existing DFTB codes. Any remaining three-center interactions needed for the calculation of condensed phase properties can be determined in $E_{r e p}$. Along these lines, we have created an environmentally dependent repulsive energy for the implicit Nbody method based on our previous three-body functional form[66], $E_{r e p}=$ $\sum_{A} \sum_{B>A} \sum_{C} V_{A B}\left(1-S_{A B C}\right) . V_{A B}$ is expressed as a ninth-order polynomial,

$$
V_{A B}=\left\{\begin{array}{cc}
\sum_{n=2}^{9} C_{n}\left(R_{c}^{A B}-R_{A B}\right)^{n}, & \left(R_{A B}<R_{c}^{A B}\right) \\
0 & \text { otherwise. }
\end{array}\right.
$$

The $C_{n}$ correspond to the polynomial coefficients and $R_{c}^{A B}$ is the pair-wise cutoff radius. $S_{A B C}$ is expressed as the multiplication of a sixth-order polynomial and hyperbolic tangent function,

$S_{A B C}=\left\{\left[\sum_{m=2}^{6} b_{m}\left(R_{c}^{A B C}-\frac{R_{A C}+R_{B C}}{2}\right)^{m}\right]_{0}^{\tanh }\left[a_{1} \exp \left(-a_{2}\left(\frac{R_{A C}+R_{B C}}{R_{A B}}\right)^{a_{3}}\right)\right], \quad\left[\left(R_{A C}+R_{B C}\right) / 2<R_{c}^{A B C}\right]\right.$

In this case, $b_{m}$ are the polynomial coefficients and $a_{1}, a_{2}$, and $a_{3}$ are also parameters to be fit. $R_{c}^{A B C}$ is the three-body interaction cutoff radius. Both pair-wise and three-body cutoff radii guarantee that $V_{A B}$ and $S_{A B C}$ will have smooth behavior around $R_{c}^{A B}$ and $R_{c}^{A B C}$, and will be equal to zero beyond. The $b_{m}$ coefficients were constrained to yield values of $S_{A B C}$ between 0 and 1 , where the function is smoothly varying. This model more closely resembles electrostatic screening methods in that the repulsive energy is completely screened and equal to zero when a third atomic site exists at the midpoint 
of the line connecting two atomic sites, and recovers a pair-wise functional form when the third atomic site is beyond $R_{c}^{A B C}$.

Determination of the repulsive energy parameters was performed using simulated annealing[69] (Table 4). We used a similar data set to our DFTBp3b optimizations[43], though here we have restricted the calculations to data points from diamond cold compression (zero Kelvin) curves up to 2064 $\mathrm{GPa}$, and snapshots of the metallic liquid at three different state points: $6.889 \mathrm{~g} / \mathrm{cc}$ and $6,852 \mathrm{~K}, 7.264 \mathrm{~g} / \mathrm{cc}$ and $9,503 \mathrm{~K}$, and $7.668 \mathrm{~g} / \mathrm{cc}$ and 15,890 K. Diamond cold curve points below $140 \mathrm{GPa}$ were computed from a Vinet equation of state fit to experimental data[70]. DFT calculations with the PBE energy functional were performed for this data set using the VASP code[71-73], with system sizes of 64 atoms, a planewave cutoff of $900 \mathrm{eV}$, and a $2 \times 2 \times 2 \mathrm{k}$-point mesh. This allowed for convergence of the total energy and stress tensor. The resulting $E_{r e p}$ yielded average deviations from the reference pressures for diamond of $\sim 4 \%$, with deviations of less than $1 \%$ for pressures above $140 \mathrm{GPa}$ (Fig. 7). We compute deviations for all liquid state points in our data set of less than $1 \%$.

Finally, we have used our repulsive energy to conduct fixed volume and temperature (NVT) MD simulations of the metallic liquid at $7.668 \mathrm{~g} / \mathrm{mL}$ and $15,890 \mathrm{~K}$, using the same k-point mesh as before and a time step of 0.5 fs. Our simulations were driven by the LAMMPS molecular software simulation suite[74], with LAMMPS also used to calculate the three-body repulsive energy and forces. The average pressure from this simulation was computed to be $1418 \mathrm{GPa}$, compared to a value of 1413 from our previous DFT-MD calculations[43]. We observe good agreement with DFT for the RDF (Fig. 8), with a coordination number for the first solvation shell of 5.5, compared to a value of 5.7 from DFT. The results for the first peak in the RDF from our implicit N-body model are slightly more structured than those from our previous DFTB-p3b fitting and from DFT-MD. However, our current model yields somewhat closer agreement for the position of the first peak as well as the second "shoulder" present at $\sim 1.7 \AA$. Further improvement in RDF prediction could conceivably be achieved through optimizing $E_{r e p}$ through force matching, (e. g., Ref.[75]), which is the subject of future work.

\section{Conclusions}

We present several new methods that include many-body effects in DFTB quantum simulations of carbon over a wide range of pressures and temperatures, including conditions where the system can experience strong electron 
delocalization and multi-nuclear center effects. Our three-center DFTB calculations with the p-orbital basis set yield an accurate description of the occupied electron states and wavefunctions for the metallic liquid state. This type of approach could prove useful for simulation of nanosecond timescale experiments, where the minimal size of the Hamiltonian matrix yields additional computational efficiency over DFTB d-orbital calculations, and the errors due to the small basis set could be swept into the optimization of the repulsive energy. Our three-center method and the implicit N-body calculations with d-orbitals allow for a more accurate description of both occupied and unoccupied electronic states up to high energy, while still providing substantial computational efficiency over standard DFT calculations. The implicit N-body Hamiltonian accounts for insulator to metal transitions such as those discussed here while requiring no modification to current DFTB implementations. Optimization of the three-body repulsive energy with this method yields an accurate description of the equation of state of both solid and liquid phases of carbon, as well as of structural properties of the liquid under very high pressures and temperatures.

All of the methods discussed here provide a general, straightforward way to extend the DFTB method to a wide variety of materials over a broad range of conditions, including geological and planetary materials such as hydrocarbons, silicon, and $\mathrm{SiO}_{2}$. The computational efficiency inherent in our models could allow for simulations of carbon-containing materials that approach the time scales of current compression experiments, where physical and chemical properties can be difficult to interrogate directly and there is historically a significant reliance on simulations for interpretation and validation of results. Long-term goals with our methods involve rapid calculation of a large number of different materials or alloys and subsequently analyzing libraries with data-mining techniques in order to make predictions for tailored properties that can be tested by experiments[76]. Bioethanol production from wood-waste sources requires development of cost effective transition metal catalysts, improved alkali metal/oxide promoters, and detailed knowledge of their effects on different supports[77]. Prevention of dendrite formation and other non-reversible processes remains a major concern for the development of future materials for rechargeable batteries[78]. Our models will have particular impact in these types of research areas, where we can overcome computationally expensive quantum methods that are generally used for interpretation of imaging and spectroscopy experiments, and prediction of properties to guide materials synthesis. 


\section{Acknowledgements}

This work was performed under the auspices of the U.S. Department of Energy by Lawrence Livermore National Laboratory under Contract DEAC52-07NA27344, and was funded by Laboratory Directed Research and Development grant \# 12-ERD-052. Computations were performed at LLNL using the Aztec, Sierra, and Cab massively parallel computers. 


\section{References}

[1] W. B. Hubbard, The interiors of giant planets, Science 214 (1981) 145.

[2] L. Segretain, G. Chabrier, M. Hernanz, E. Garcia-Berro, J. Isern, R. Mochkovitch, Cooling theory of crystallized white dwarfs, Astrophys. J. 434 (1994) 641.

[3] S. Seager, M. Kuchner, C. A. Hier-Majumder, B. Militzer, Mass-radius relationships for solid exoplanets, Astrophys. J. 669 (2007) 1279.

[4] S. Haan, M. Herrmann, J. Salmonson, P. Amendt, D. Callahan, T. Dittrich, M. Edwards, O. Jones, M. Marinak, D. Munro, S. Pollaine, B. Spears, L. Suter, Update on design simulations for nif ignition targets, and the rollup of all specifications into an error budget, Euro. Phys. J. D 44 (2007) 249-258.

[5] M. R. Manaa, E. J. Reed, L. E. Fried, N. Goldman, Nitrogen-rich heterocycles as reactivity retardants in shocked insensitive explosives, J. Am. Chem. Soc. 131 (2009) 5493.

[6] N. Goldman, E. J. Reed, L. E. Fried, I.-F. W. Kuo, A. Maiti, Synthesis of glycine-containing complexes in impacts of comets on early earth, Nat. Chem. 2 (2010) 949.

[7] Z. Martins, M. C. Price, N. Goldman, M. A. Sephton, M. J. Burchell, Shock synthesis of organics from simple ice mixtures, Nat. Geosci. 6 (2013) 1045.

[8] N. Goldman, I. Tamblyn, Prebiotic chemistry within a simple impacting icy mixture, The Journal of Physical Chemistry A 117 (5124) (2013) $5124-5131$.

[9] A. F. Goncharov, V. V. Struzkhin, M. S. Somayazulu, R. J. Hemley, H. K. Mao, Compression of ice to 210 gigapascals: Infrared evidence for a symmetric hydrogen-bonded phase, Science 273 (1996) 218.

[10] A. F. Goncharov, N. Goldman, L. E. Fried, J. C. Crowhurst, I.-F. W. Kuo, C. J. Mundy, J. M. Zaug, Dynamic ionization of water under extreme conditions, Phys. Rev. Lett. 94 (12) (2005) 125508.

[11] D. K. Bradly, J. H. Eggert, R. F. Smith, S. T. Prisbrey, D. G. Hicks, D. G. Braun, J. Biener, A. V. Hamza, R. E. Rudd, G. W. Collins, Diamond at 800 gpa, Phys. Rev. Lett. 102 (2009) 075503. 
[12] D. K. Bradley, J. H. Eggert, D. Hicks, P. Celliers, S. J. Moon, R. C. Cauble, G. Collins, Shock compressing diamond to a conducting fluid, Phys. Rev. Lett. 93 (2004) 195506.

[13] H. Nagao, K. G. Nakamura, K. Kondo, N. Ozaki, K. Takamatsu, T. Ono, T. Shiota, D. Ichinose, K. A. Tanaka, K. Wakabayashi, K. Okada, M. Yoshida, M. Nakai, K. Nagai, K. Shigemori, T. Sakaiya, K. Otani, Hugoniot measurement of diamond under laser shock compression up to 2 tpa, Phys. Plasmas 13 (2006) 052705.

[14] D. G. Hicks, T. R. Boehly, P. M. Celliers, D. K. Bradley, J. H. Eggert, R. S. McWilliams, R. Jeanloz, G. W. Collins, High-precision measurements of the diamond hugoniot in and above the melt region, Phys. Rev. Lett. 78 (2008) 174102.

[15] A. A. Correa, S. A. Bonev, G. Galli, Carbon under extreme conditions: Phase boundaries and electronic properties from first-principles theory, Proceedings of the National Academy of Sciences of the United States 103 (5) (2006) 1204.

[16] M. D. Knudson, M. P. Desjarlais, D. H. Dolan, Shock-wave exploration of the high-pressure phases of carbon, Science 322 (2008) 1822.

[17] G. A. Lyzenga, T. J. Ahrens, W. J. Nellis, A. C. Mitchell, The temperature of shock-compressed water, J. Chem. Phys. 76 (1982) 6282.

[18] M. A. Barrios, D. G. Hicks, T. R. Boehly, D. E. Fratanduono, J. H. Eggert, P. M. Celliers, G. W. Collins, D. D. Meyerhofer, High-precision measurements of the equation of state of hydrocarbons at 1-10 Mbar using laser-driven shock waves, Phys. Plasmas 17 (2010) 056307.

[19] K. K. M. Lee, L. R. Benedetti, R. Jeanloz, P. M. Celliers, J. H. Eggert, D. G. Hicks, S. J. Moon, A. Mackinnon, L. B. D. Silva, D. K. Bradley, W. Unites, G. W. Collins, Laser-driven shock experiments on precompressed water: Implications for "icy" giant planets, J. Chem. Phys. 125 (2006) 014701.

[20] A. A. Correa, L. X. Benedict, D. A. Young, E. Schwegler, S. Bonev, First-principles multiphase equation of state of carbon under extreme conditions, Phys. Rev. B 78 (2008) 024101.

[21] J. H. Eggert, D. G. Hicks, P. M. Celliers, D. K. Bradley, R. S. McWilliams, R. Jeanloz, J. E. Miller, T. R. Boehly, G. Collins, Melting temperature of diamond at ultrahigh pressure, Nat. Phys. 6 (2010) 40. 
[22] J. Sun, D. D. Klug, R. Martoňák, Structural transformations in carbon under extreme pressure: Beyond diamond, J. Chem. Phys. 130 (2009) 194512.

[23] L. Fried, W. M. Howard, Explicit gibbs free energy equation of state applied to the carbon phase diagram, Phys. Rev. B. 61 (13) (2000) 8734.

[24] N. Goldman, R. S. Fellers, M. G. Brown, L. B. Braly, C. J. Keoshian, C. Leforestier, R. J. Saykally, Spectroscopic determination of the water dimer intermolecular potential-energy surface, J. Chem. Phys. 116 (23) (2002) 10148.

[25] N. Goldman, C. Leforestier, R. J. Saykally, A 'first principles' potential energy surface for liquid water from VRT spectroscopy of water clusters, Phil. Trans. R. Soc. A 363 (1827) (2005) 493-508.

[26] P. Hohenberg, W. Kohn, Inhomogeneous electron gas, Phys. Rev. B 136 (1964) 864.

[27] W. Kohn, L. Sham, Self-consistent equations including exchange and correlation effects, Phys. Rev. A 140 (1965) 1133.

[28] E. Schwegler, M. Sharma, F. Gygi, G. Galli, Melting of ice under pressure, Proc. Nat. Acad. Sci.(USA) 105 (2008) 14779.

[29] A. F. Goncharov, C. Sanloup, N. Goldman, J. C. Crowhurst, N. Guignot, M. Mezouar, Y. Meng, Dissociative melting of ice vii at high pressure, J. Chem. Phys. 130 (2009) 124514.

[30] N. Goldman, L. E. Fried, I.-F. W. Kuo, C. J. Mundy, Bonding in the superionic phase of water, Phys. Rev. Lett. 94 (21) (2005) 217801.

[31] N. Goldman, E. J. Reed, I.-F. W. Kuo, L. E. Fried, C. J. Mundy, A. Curioni, Ab initio simulation of the equation of state and kinetics of shocked water, J. Chem. Phys. 130 (2009) 124517.

[32] N. Goldman, E. J. Reed, L. E. Fried, Quantum corrections to shock hugoniot temperatures, J. Chem. Phys. 131 (2009) 204103.

[33] C. J. Mundy, A. Curioni, N. Goldman, I.-F. Kuo, E. Reed, L. E. Fried, M. Ianuzzi, Ultarfast transformation of graphite into diamond: An $a b$ initio study of graphite under shock compression, J. Chem. Phys. 128 (18) (2008) 184701. 
[34] C. Wu, L. E. Fried, L. H. Yang, N. Goldman, S. Bastea, Catalytic behaviour of dense hot water, Nat. Chem. 1 (2009) 57.

[35] T. R. Mattsson, J. M. D. Lane, K. R. Cochrane, M. P. Desjarlais, A. P. Thompson, F. Pierce, G. S. Grest, First-principles and classical molecular dynamics simulation of shocked polymers, Phys. Rev. B 81 (2010) 054103.

[36] S. Root, T. A. Haill, J. M. D. Lane, A. P. Thompson, G. S. Grest, D. G. Schoen, T. R. Mattsson, Shock compression of hydrocarbon foam to 200 GPa: Experiments, atomistic simulations, and mesoscale hydrodynamic modeling., Journal of Applied Physics 114 (2013) 103502.

[37] M. Elstner, D. Porezag, G. Jungnickel, J. Elsner, M. Haugk, T. Frauenheim, S. Suhai, G. Seifert, Self-consistent-charge density-functional tight-binding method for simulations of complex materials properties, Phys. Rev. B 58 (1998) 7260.

[38] N. Goldman, S. Bastea, Nitrogen oxides as a chemistry trap in detonating oxygen-rich materials, J. Phys. Chem. A 118 (2014) 2897-2903.

[39] A. B. Kunz, Three-center corrections to the $\mathrm{NaCl}$ valence band, Phys. Rev. 162 (1967) 789.

[40] O. F. Sankey, D. J. Niklewski, Ab initio multicenter tight-binding model for molecular-dynamics simulations and other applications in covalent systems, Phys. Rev. B 40 (1989) 3979.

[41] M.-H. Tsai, O. F. Sankey, J. D. Dow, Charge transfer in ab initio realspace molecular dynamics, Phys. Rev. B 46 (1992) 10464.

[42] M. S. Tang, C. Z. Wang, C. T. Chan, K. M. Ho, Environment-dependent tight-binding model, Phys. Rev. B 53 (1996) 979.

[43] N. Goldman, S. G. Srinivasan, S. Hamel, L. E. Fried, M. Gaus, M. Elstner, Determination of a density functional tight binding model with an extended basis set and three-body repulsion for carbon under extreme pressures and temperatures, J. Phys. Chem. C 117 (2013) 7885 - 7894,

[44] J. P. Lewis, P. Jelínek, J. Ortega, A. A. Demkov, D. G. Trabada, B. Haycock, H. Wang, G. Adams, J. K. Tomfohr, E. Abad, H. Wang, D. A. Drabold, Advances and applications in the fireball ab initio tightbinding molecular-dynamics formalism, Phys. Status Solidi B 248 (9) (2011) 1989-2007. 
[45] Y.-Z. Jin, Y.-C. Wang, Z.-Y. Geng, H.-J. Wang, Y.-Z. Gan, Competitive activation of ch and of bonds in gas phase reaction of $\mathrm{Ir}^{+}$with $\mathrm{CH}_{3} \mathrm{~F}$ : A DFT study, Journal of Organometallic Chemistry 717 (0) (2012) 195 $-201$.

[46] E. J. Reed, M. R. Manaa, L. E. Fried, K. R. Glaesemann, J. D. Joannopoulos, A transient semi-metallic layer in detonating nitromethane, Nature Physics 4 (2008) 72-76.

[47] W. M. Foulkes, R. Haydock, Tight-binding models and densityfunctional theory, Phys. Rev. B 39 (17) (1989) 12520.

[48] R. E. Cohen, M. J. Mehl, D. A. Papaconstantopoulos, Tight-binding total-energy method for transition and noble metals, Phys. Rev. B 50 (1994) 14694.

[49] H. Haas, C. Z. Wang, M. Fähnle, C. Elsässer, K. M. Ho, Environmentdependent tight-binding model for molybdenum, Phys. Rev. B 57 (1998) 1461.

[50] D. A. Areshkin, O. A. Shenderova, J. D. Schall, S. P. Adiga, D. W. Brenner, A self-consistent tight binding model for hydrocarbon systems: application to quantum transport simulation, J. Phys.: Condens. Matter 16 (2004) 6851-6866.

[51] M. Gaus, Q. Cui, M. Elstner, DFTB3: Extension of the self-consistentcharge density-functional tight-binding method (SCC-DFTB), J. Chem. Theory Comput. 7 (2011) 931.

[52] M. Gaus, A. Goez, M. Elstner, Parametrization and benchmark of DFTB3 for organic molecules, Journal of Chemical Theory and Computation 9 (2012) 338-354.

[53] S. G. Srinivasan, N. Goldman, I. Tamblyn, S. Hamel, M. Gaus, Determination of a density functional tight binding model with an extended basis set and three-body repulsion for hydrogen under extreme thermodynamic conditions, J. Phys. Chem. A 118 (2014) 5520-5528.

[54] N. Ileri, N. Goldman, Graphene and nano-diamond synthesis in expansions of molten liquid carbon, J. Chem. Phys 141 (2014) 164709.

[55] T. Frauenheim, G. Seifert, M. Elstner, T. Niehaus, C. Köhler, M. Amkreutz, M. Sternberg, Z. Hajnal, A. D. Carlo, S. Suhai, Atom- 
istic simulations of complex materials: ground-state and excited-state properties, Journal of Physics: Condensed Matter 14 (2002) 3015-3047.

[56] P. Koskinen, V. Mäkinen, Density-functional tight-binding for beginners, Comp. Mater. Sci. 47 (2009) 237.

[57] D. Porezag, T. Frauenheim, T. Köhler, G. Seifert, R. K. Kaschner, Construction of tight-binding-like potentials on the basis of density functional theory: Application to carbon, Phys. Rev. B 51 (1995) 12947.

[58] G. Seifert, Tight-binding density functional theory: An approximate kohn-sham dft scheme, J. Phys. Chem. A 111 (5609-5613).

[59] J. M. Soler, E. Artacho, J. D. Gale, A. García, J. Junquera, P. Ordejón, D. Sánchez-Portal, The siesta method for ab initio order-N materials simulation, Journal of Physics: Condensed Matter 14 (11) (2002) 2745.

[60] N. Troullier, J. L. Martins, Efficient pseudopotentials for plane-wave calculations, Phys.Rev. B 43 (1991) 1993.

[61] J. P. Perdew, K. Burke, M. Ernzerhof, Generalized gradient approximation made simple, Phys. Rev. Lett. 78 (7) (1997) 1396 - 1396.

[62] D. Nguyen-Manh, D. G. Pettifor, V. Vitek, Analytic environmentdependent tight-binding bond integrals: Application to $\mathrm{MoSi}_{2}$, Phys. Rev. Lett. 85 (19) (2000) 4136.

[63] M. Mrovec, D. Nguyen-Manh, D. G. Pettifor, V. Vitek, Bond-order potential for molybdenum: Application to dislocation behavior, Phys. Rev. B 69 (9) (2004) 094115.

[64] B. Aradi, B. Hourahine, T. Frauenheim, DFTB+, a sparse matrix-based implementation of the dftb method, J. Phys. Chem. A 111 (2007) 5678, http://www.dftb-plus.info.

[65] N. D. Mermin, Thermal properties of the inhomogenous electron gas, Phys. Rev. 137 (5A) (1965) 1441.

[66] N. Goldman, L. E. Fried, Extending the density functional tight binding method to carbon under extreme conditions, J. Phys. Chem. C 116 (2012) 2198.

[67] J. Dong, D. A. Drabold, Atomistic structure of band-tail states in amorphous silicon, Phys. Rev. Lett. 80 (9) (1998) 1928-1931. 
[68] T. R. Mattsson, M. P. Desjarlais, Phase diagram and electrical conductivity of high energy-density water from density functional theory, Phys. Rev. Lett. 97 (2006) 017801.

[69] W. H. Press, S. A. Teukolsky, W. T. Vetterling, B. P. Flannery, Numerical Recipes in Fortran 77, Cambridge University Press, Cambridge, 1992.

[70] F. Occelli, P. Loubeyre, R. Letoullec, Properties of diamond under hydrostatic pressures up to 140 gpa, Nat. Mater. 2 (2003) 151.

[71] G. Kresse, J. Hafner, Ab initio molecular dynamics for liquid metals, Phys. Rev. B 47 (1993) R558.

[72] G. Kresse, J. Hafner, Ab initio molecular dynamics simulation of the liquid-metal-amorphous-semiconductor transition in germanium, Phys. Rev. B 49 (1994) 14251.

[73] G. Kresse, J. Furthmüller, Efficient iterative schemes for ab initio totalenergy calculations using a plane-wave basis set, Phys. Rev. B 54 (1996) 11169 .

[74] S. Plimpton, Fast parallel algorithms for short-range molecular dynamics, J. Comp. Phys. 117 (1995) 1, http://lammps.sandia.gov.

[75] S. Izvekov, M. Parrinello, C. J. Burnham, G. A. Voth, Effective force fields for condensed phase systems from ab initio molecular dynamics simulation: A new method for force-matching, J. Chem. Phys. 120 (2004) 10896.

[76] C. C. Fischer, K. J. Tibbetts, D. Morgan, G. Ceder, Predicting crystal structure by merging datamining with quantummechanics, Nat. Mater. 5 (2006) 641.

[77] A. Egbebi, V. Schwartz, S. H. Overbury, J. J. Spivey, Effect of Li promoter on titania-supported Rh catalyst for ethanol formation from $\mathrm{CO}$ hydrogenation, Catalysis Today 149 (2010) 91.

[78] C. M. López, K.-S. Choi, Electrochemical synthesis of dendritic zinc films composed of systematically varying motif crystals, Langmuir 22 (2006) 10625-10629. 


\section{Figures}

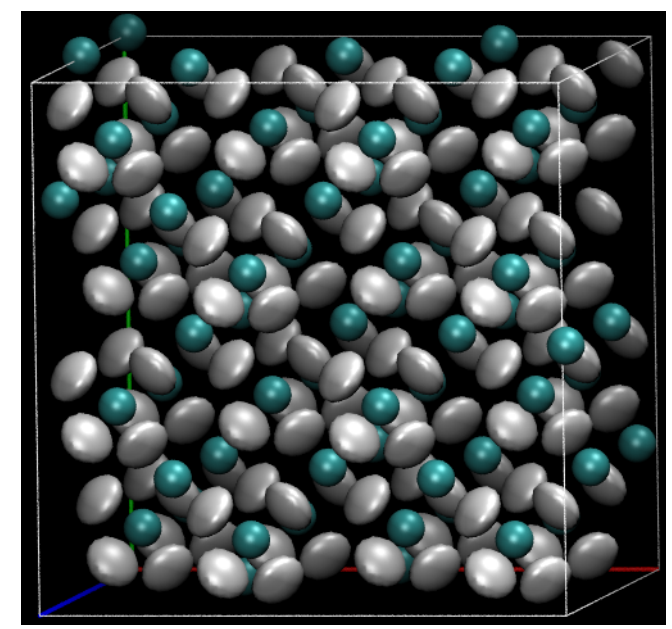

(a) $3.51 \mathrm{~g} / \mathrm{mL}, 300 \mathrm{~K}$

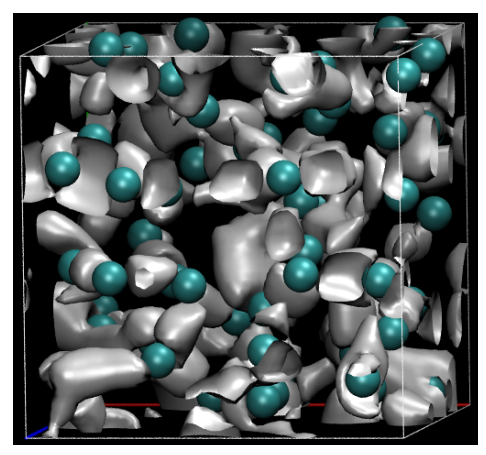

(b) $7.668 \mathrm{~g} / \mathrm{mL}, 15,890 \mathrm{~K}$

Figure 1: Plot of an isosurface of the electron density of diamond (a) and metallic liquid carbon (b), with the atomic valence charge densities subtracted out for clarity. Carbon atoms are shown as light blue spheres. The smaller size of (b) corresponds to the higher density of the system. The diamond configuration exhibits electron density localized between atoms, whereas the metallic liquid configuration shows a high degree of electronic delocalization across multiple atomic sites. 


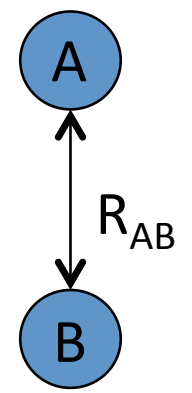

(a)

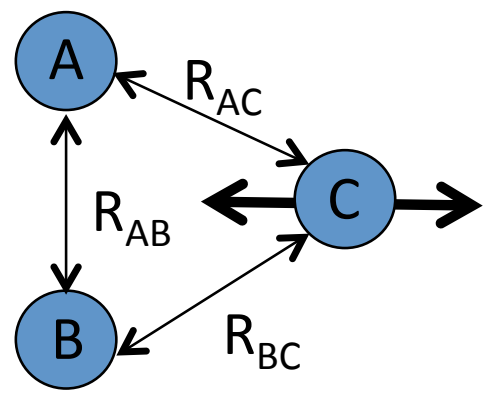

(b)

Figure 2: Dimer and trimer geometries included in determination of the onsite (a) and offsite (b) matrix elements. Dimer geometries were sampled along the $R_{A B}$-axis. For the trimer geometries, position of the $C$-atom was varied along the bold arrow. 


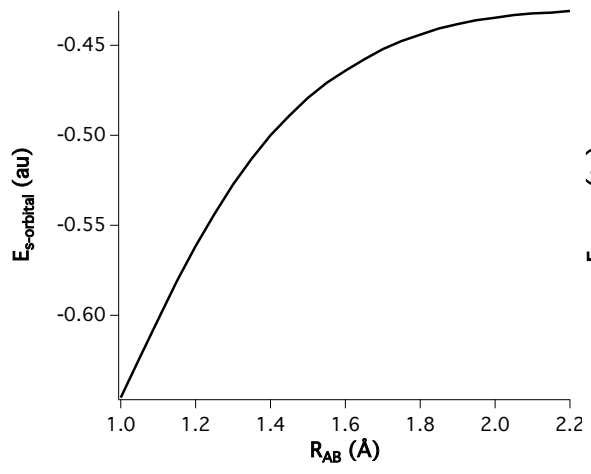

(a) s-orbital energies

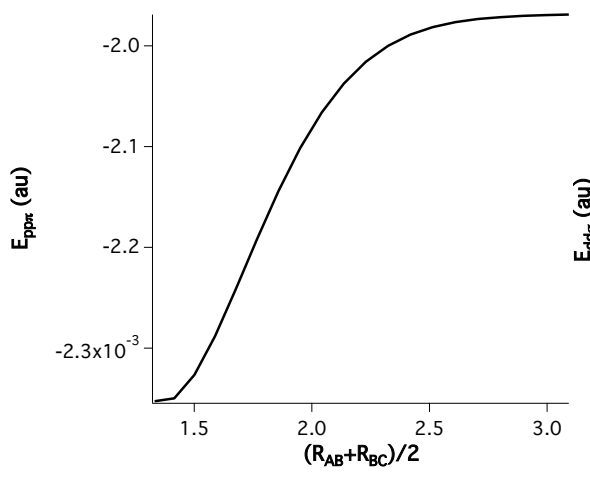

(c) $\mathrm{pp} \pi$ interactions

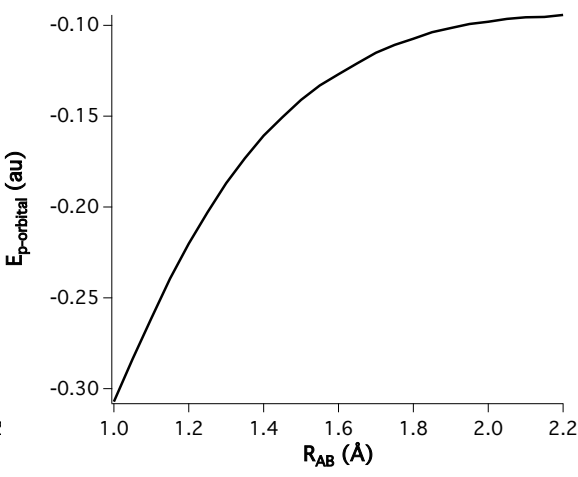

(b) p-orbital energies

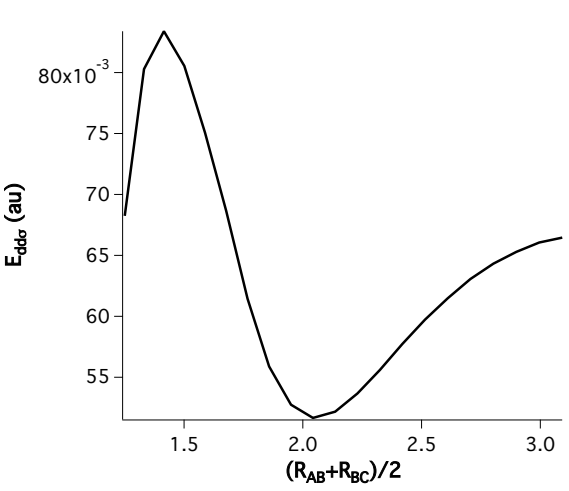

(d) $\mathrm{dd} \sigma$ interactions

Figure 3: Examples of the onsite and offsite matrix elements as a function of interatomic distance. In general, we found that both onsite and offsite matrix elements varied monotonically, excluding the $\mathrm{dd} \sigma$ interactions which have a higher degree of angular character. 


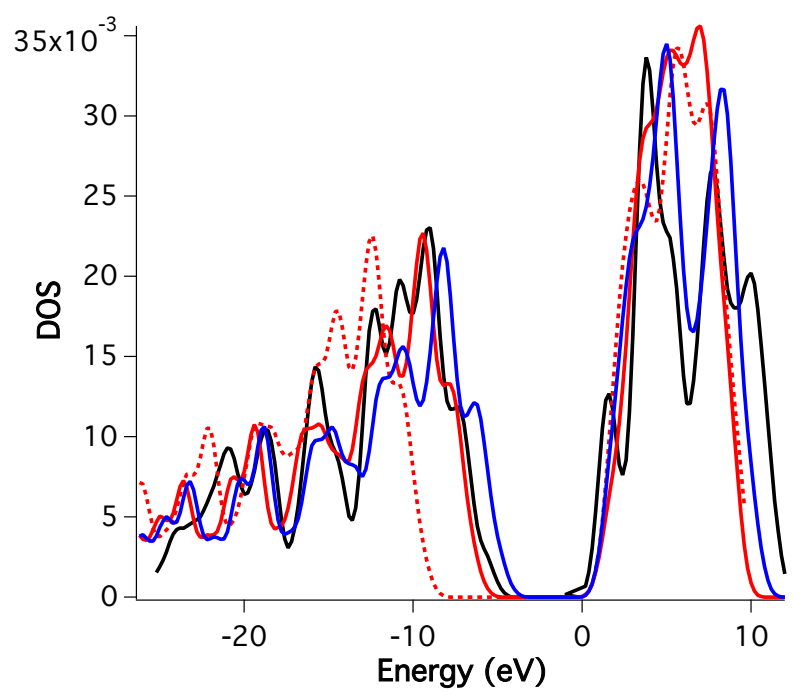

Figure 4: Electronic density of states plots for diamond at $300 \mathrm{~K}, 3.51 \mathrm{~g} / \mathrm{mL}$. All curves have been shifted to set the Fermi energy equal to zero. The dashed red curve corresponds to result from the $3 \mathrm{~b}-\mathrm{p}$ model, the solid red curve to $3 \mathrm{~b}-\mathrm{d}$, the solid blue curve to the implicit N-body method, and the solid black curve to DFT-SZP. Results from standard DFTB as well as DFT-SZ were omitted for the sake of clarity. 
Figure 5: Electronic density of states plots of the metallic liquid phase at $7.668 \mathrm{~g} / \mathrm{mL}$ and $15,890 \mathrm{~K}$, using a p-orbital or d-orbital basis set. All curves have been shifted to set the Fermi energy equal to zero. For each plot, the dashed blue curve corresponds to results from standard DFTB, the solid red curve to our three-body Hamiltonian (3b-p or 3b-d), and the solid black curve to results from DFT-SZ or DFT-SZP. In the case of the d-orbital calculation, the solid blue line corresponds to the result from our implicit N-body method. 


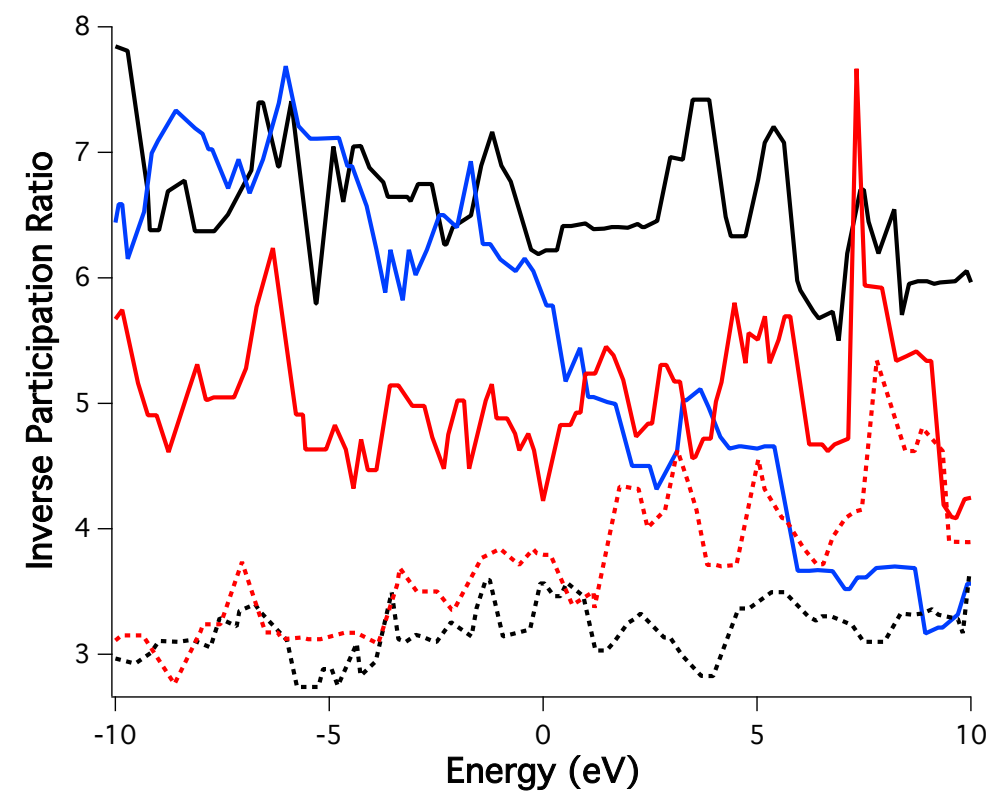

Figure 6: Inverse participation ratios for eigenfunctions near the Fermi energy (shifted to $0 \mathrm{eV}$ ). The solid black line corresponds to the result from DFT-SZP, the solid red line to the 3b-d model, the solid blue line to the implicit N-body method, the dashed black line to DFT-SZ, and the dashed red line to $3 \mathrm{~b}-\mathrm{p}$. 


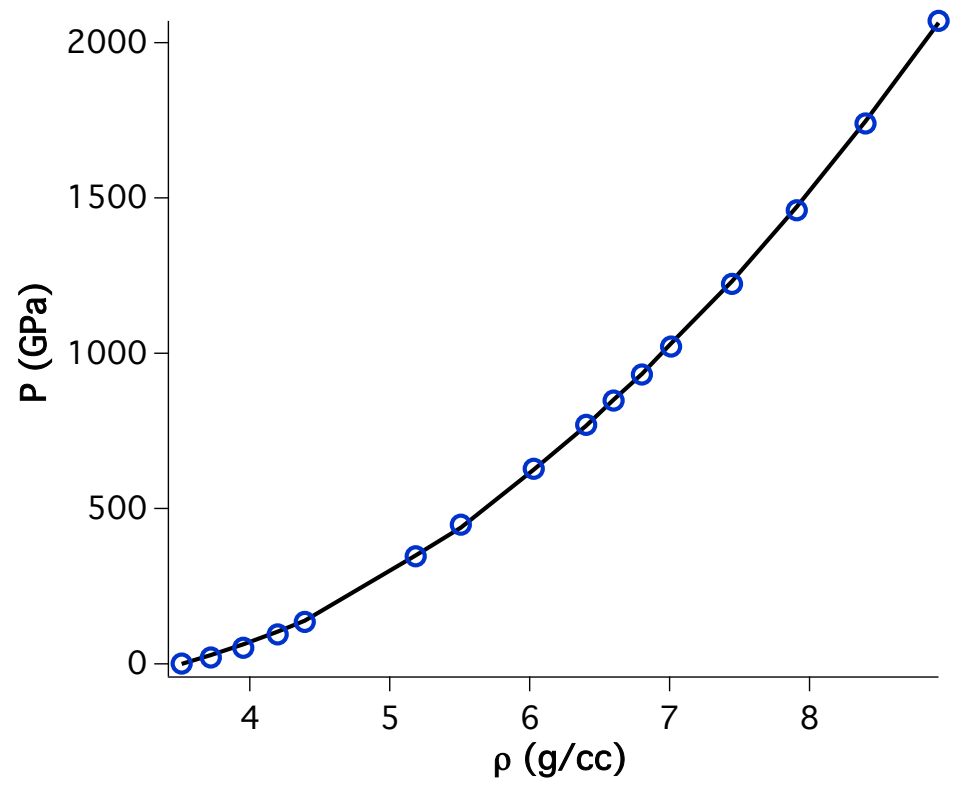

Figure 7: Diamond cold curve calculation. The solid black line corresponds to results from the experimental EOS for $\mathrm{P} \leq 140 \mathrm{GPa}$, and to DFT beyond. The blue open circles correspond to results from our repulsive energy optimization. 


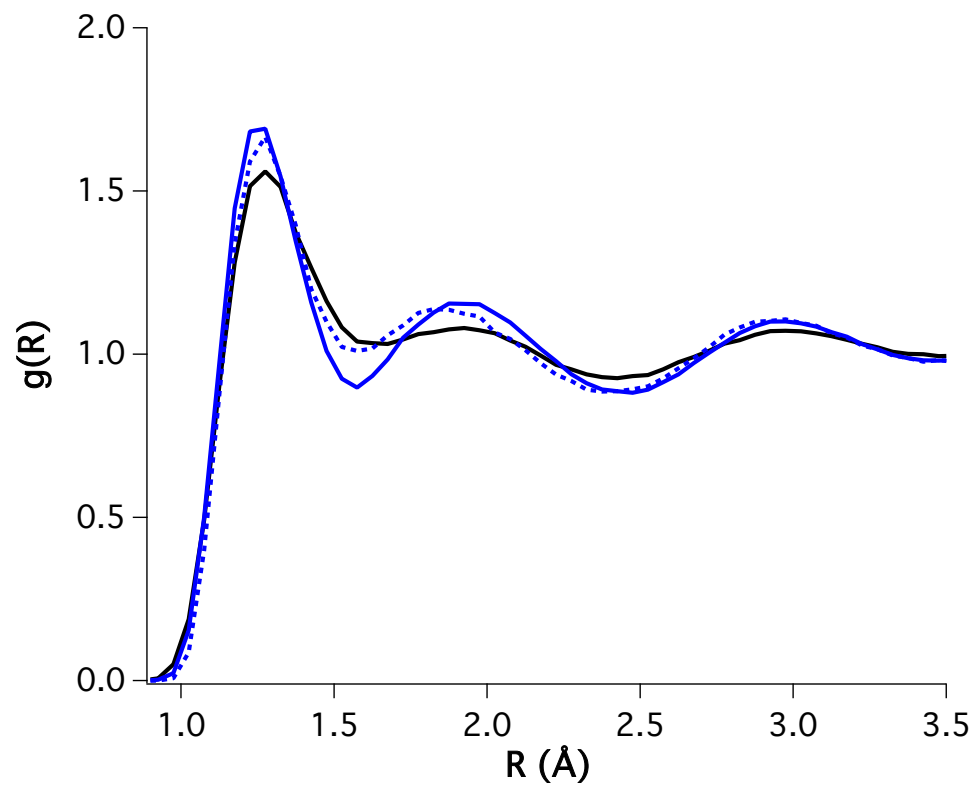

Figure 8: Radial distribution function for metallic liquid carbon at $7.668 \mathrm{~g} / \mathrm{mL}$ and 15,890 K. The solid black line corresponds to previously computed results from DFT-PBE, the solid blue line to results for our repulsive energy optimization with the implicit N-body Hamiltonian, and the dashed blue line to our previous fitting of DFTB-p3b. 
Table 1: Parameters for the radial functions representing the environmental dependence of the onsite matrix elements. All parameters are in atomic units. The cutoff radius was set to 2.93 au $(1.55 \AA)$ for all three orbitals. Each pair-wise radial function was scaled by an additional factor of 0.6 as mentioned in the text.

\begin{tabular}{cccc}
\hline parameter & s-orbital & p-orbital & d-orbital \\
\hline \hline$\alpha_{2}$ & -2.06510 & -6.78063 & 1.35167 \\
$\alpha_{3}$ & 7.23809 & 23.34945 & -5.14759 \\
$\alpha_{4}$ & -12.62057 & -40.29283 & 8.84578 \\
$\alpha_{5}$ & 10.21010 & 32.31505 & -7.12563 \\
$\alpha_{6}$ & -3.11284 & -9.81561 & 2.18164
\end{tabular}

Table 2: Orbital energies used for the explicit three-center and the implicit N-body calculations.

\begin{tabular}{ccc}
\hline orbital energy & three-center & implicit N-body \\
\hline \hline$E_{s}$ & -0.50489172 & -0.47084563 \\
$E_{p}$ & -0.19435511 & -0.13324365 \\
$E_{d}$ & 0.8 & 0.5
\end{tabular}

Table 3: Parameters for the environmental dependence of the offsite matrix elements. Values for the $\mathrm{dd} \pi$ and $\mathrm{dd} \delta$ Slater-Koster interactions were set to be equal in this study.

\begin{tabular}{cccc}
\hline offsite interaction & $\beta_{1}$ & $\beta_{2}$ & $\beta_{3}$ \\
\hline \hline $\operatorname{ss} \sigma$ & 1.49281 & 1.20786 & 1.54175 \\
$\operatorname{sp} \sigma$ & 4.54728 & 1.99099 & 1.18470 \\
$\operatorname{sd} \sigma$ & 9004.81 & 8.71421 & 0.47413 \\
$\operatorname{pp} \sigma$ & 122.858 & 4.73905 & 0.69380 \\
$\operatorname{pp} \pi$ & 0.66979 & 0.15893 & 3.51410 \\
$\operatorname{pd} \sigma$ & 64.9761 & 3.39154 & 1.41462 \\
$\operatorname{pd} \pi$ & 14.1249 & 1.41502 & 1.50546 \\
$\mathrm{dd} \sigma$ & 6.04710 & 1.97766 & 1.61866 \\
$\mathrm{dd} \pi$ & 0.59704 & 0.17163 & 3.75520 \\
$\mathrm{dd} \delta$ & 0.59704 & 0.17163 & 3.75520
\end{tabular}


Table 4: Parameters for the pairwise and three-body components of the repulsive energy.

\begin{tabular}{cccc}
\hline$V_{A B}$ & Value $(\mathrm{au})$ & $S_{A B C}$ & Value $(\mathrm{au})$ \\
\hline \hline$C_{2}$ & -0.34926622 & $b_{2}$ & 1.43845021 \\
$C_{3}$ & 3.15472606 & $b_{3}$ & -1.03775491 \\
$C_{4}$ & -9.25908482 & $b_{4}$ & -0.25492344 \\
$C_{5}$ & 14.08975165 & $b_{5}$ & -1.62059276 \\
$C_{6}$ & -12.02282535 & $b_{6}$ & -1.01728843 \\
$C_{7}$ & 5.87188900 & $a_{1}$ & 24.99999661 \\
$C_{8}$ & -1.53090452 & $a_{2}$ & 0.39899962 \\
$C_{9}$ & 0.16682527 & $a_{3}$ & 3.39000040 \\
$R_{c}^{A B}$ & 3.47002482 & $R_{c}^{A B C}$ & 3.79993328
\end{tabular}


Nir Goldman received a B.S. in Chemistry from Yale University in 1997 and a Ph.D. in Physical Chemistry from the University of California, Berkeley, in 2003. He then joined Lawrence Livermore National Laboratory (LLNL) as a post-doctoral researcher, where he was promoted to the position of staff scientist in 2006. His current research interests involve the development of novel approaches to quantum mechanical simulations of chemical reactivity within condensed matter, including materials under extremely high pressures and temperatures and the astrobiological synthesis of life-building compounds under extreme thermodynamic conditions. 
*Author Photos

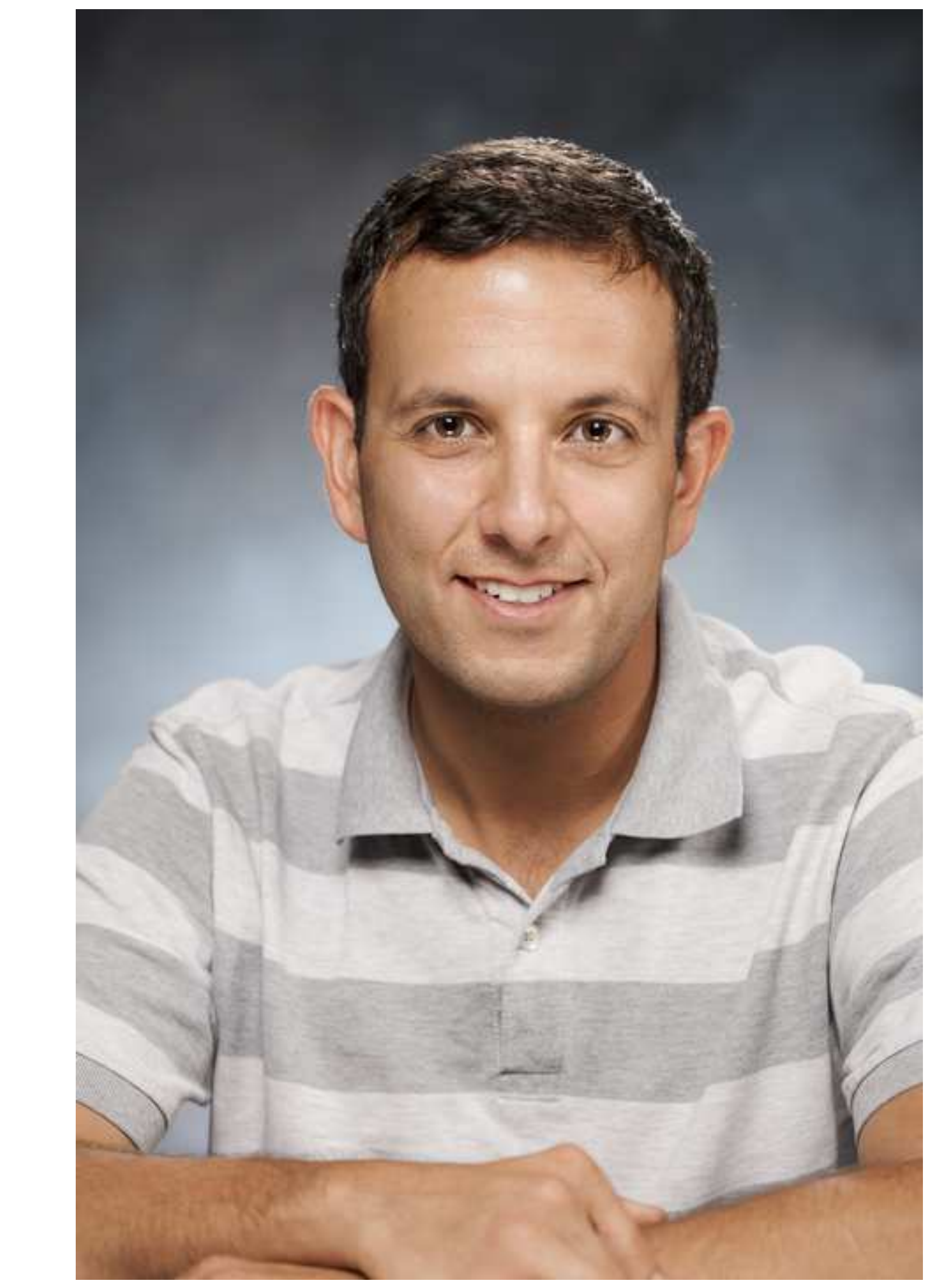

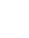

\section{Author Photos}

s

the

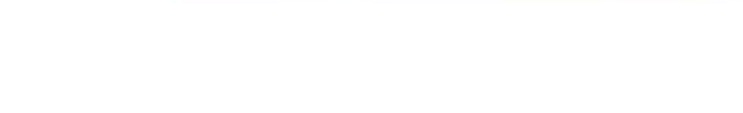




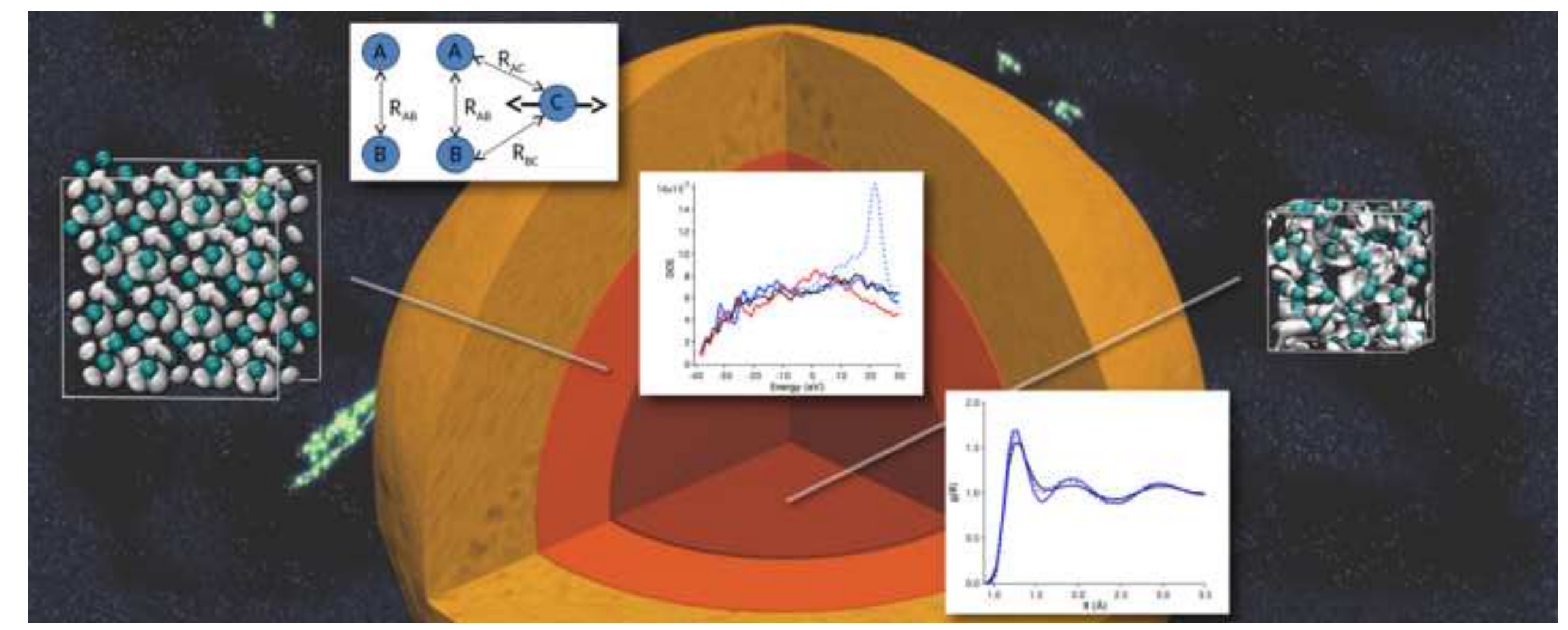

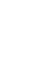

